\title{
Series acceleration formulas for beta values
}

\author{
Kh. Hessami Pilehrood and T. Hessami Pilehrood
}

Mathematics Department, Faculty of Basic Sciences, Shahrekord University, Shahrekord, P.O. Box 115, Iran.

received Sep. 13, 2009, revised March 17, 2010, accepted March 31, 2010.

We prove generating function identities producing fast convergent series for the sequences $\beta(2 n+1), \beta(2 n+2)$ and $\beta(2 n+3)$, where $\beta$ is Dirichlet's beta function. In particular, we obtain a new accelerated series for Catalan's constant convergent at a geometric rate with ratio $2^{-10}$, which can be considered as an analog of Amdeberhan-Zeilberger's series for $\zeta(3)$.

Keywords: Dirichlet's beta function, Catalan's constant, Apéry-like series, generating function, convergence acceleration, Markov-Wilf-Zeilberger method, Markov-WZ pair.

In honor of P. Flajolet's 60th birthday

\section{Introduction}

In this paper we continue our study on finding generalized identities [8, 9] that produce fast convergent series for some classical constants. In 1978, R. Apéry used the fast convergent series

$$
\zeta(3)=\frac{5}{2} \sum_{k=1}^{\infty} \frac{(-1)^{k-1}}{k^{3}\left(\begin{array}{c}
2 k \\
k
\end{array}\right)}
$$

first obtained by A. A. Markov [14] to derive the irrationality of $\zeta(3)$ [17]. This series converges at a geometric rate with ratio $1 / 4$. A general formula giving analogous series for all $\zeta(2 n+3), n \geq 0$, was proved by Koecher [11] (and independently in an expanded form by Leshchiner [12]). For $|a|<1$, it has the form

$$
\sum_{n=0}^{\infty} \zeta(2 n+3) a^{2 n}=\sum_{k=1}^{\infty} \frac{1}{k\left(k^{2}-a^{2}\right)}=\frac{1}{2} \sum_{k=1}^{\infty} \frac{(-1)^{k-1}}{k^{3}\left(\begin{array}{c}
2 k \\
k
\end{array}\right)} \frac{5 k^{2}-a^{2}}{k^{2}-a^{2}} \prod_{m=1}^{k-1}\left(1-\frac{a^{2}}{m^{2}}\right)
$$

Expanding the right-hand side of (2) by powers of $a^{2}$ and comparing coefficients of $a^{2 n}$ on both sides leads to the Apéry-like series for $\zeta(2 n+3)$. In particular, comparing constant terms $(n=0)$ recovers 1365-8050 @ 2010 Discrete Mathematics and Theoretical Computer Science (DMTCS), Nancy, France 
Markov's formula (1) and comparing coefficients of $a^{2}$ and $a^{4}$ gives the following two formulas:

$$
\begin{aligned}
& \zeta(5)=2 \sum_{k=1}^{\infty} \frac{(-1)^{k+1}}{k^{5}\left(\begin{array}{c}
2 k \\
k
\end{array}\right)}-\frac{5}{2} \sum_{k=1}^{\infty} \frac{(-1)^{k+1}}{k^{3}\left(\begin{array}{c}
2 k \\
k
\end{array}\right)} \sum_{j=1}^{k-1} \frac{1}{j^{2}}, \\
& \zeta(7)=2 \sum_{k=1}^{\infty} \frac{(-1)^{k+1}}{k^{7}\left(\begin{array}{c}
2 k \\
k
\end{array}\right)}-2 \sum_{k=1}^{\infty} \frac{(-1)^{k+1}}{k^{5}\left(\begin{array}{c}
2 k \\
k
\end{array}\right)} \sum_{j=1}^{k-1} \frac{1}{j^{2}}+\frac{5}{2} \sum_{k=1}^{\infty} \frac{(-1)^{k+1}}{k^{3}\left(\begin{array}{c}
2 k \\
k
\end{array}\right)} \sum_{m=1}^{k-1} \frac{1}{m^{2}} \sum_{j=1}^{m-1} \frac{1}{j^{2}},
\end{aligned}
$$

respectively. In 1996, inspired by this result, J. Borwein and D. Bradley [4] applied extensive computer searches on the base of integer relations algorithms looking for additional zeta identities of this sort. This led to the discovery of the new identity

$$
\zeta(7)=\frac{5}{2} \sum_{k=1}^{\infty} \frac{(-1)^{k+1}}{k^{7}\left(\begin{array}{c}
2 k \\
k
\end{array}\right)}+\frac{25}{2} \sum_{k=1}^{\infty} \frac{(-1)^{k+1}}{k^{3}\left(\begin{array}{c}
2 k \\
k
\end{array}\right)} \sum_{j=1}^{k-1} \frac{1}{j^{4}},
$$

which is simpler than Koecher's formula for $\zeta(7)$, and similar identities for $\zeta(9), \zeta(11), \zeta(13)$, etc. This allowed them to conjecture that certain of these identities, namely those for $\zeta(4 n+3)$ are given by the following generating function formula [3]:

$$
\sum_{n=0}^{\infty} \zeta(4 n+3) a^{4 n}=\sum_{k=1}^{\infty} \frac{k}{k^{4}-a^{4}}=\frac{5}{2} \sum_{k=1}^{\infty} \frac{(-1)^{k+1} k}{\left(\begin{array}{c}
2 k \\
k
\end{array}\right)\left(k^{4}-a^{4}\right)} \prod_{m=1}^{k-1}\left(\frac{m^{4}+4 a^{4}}{m^{4}-a^{4}}\right), \quad|a|<1 .
$$

The validity of (4) was proved later by G. Almkvist and A. Granville [1] in 1999. Expanding the right-hand side of (4) in powers of $a^{4}$ gives a formula for $\zeta(4 n+3)$, which for $n \geq 1$ contains fewer summations the the corresponding formula generated by (2). In particular, comparing constant terms gives (1) and comparing coefficients of $a^{4}$ yields 3 .

There exists a bivariate unifying formula for identities (2) and (4)

$$
\sum_{k=1}^{\infty} \frac{k}{k^{4}-x^{2} k^{2}-y^{4}}=\frac{1}{2} \sum_{k=1}^{\infty} \frac{(-1)^{k+1}}{k\left(\begin{array}{c}
2 k \\
k
\end{array}\right)} \frac{5 k^{2}-x^{2}}{k^{4}-x^{2} k^{2}-y^{4}} \prod_{m=1}^{k-1} \frac{\left(m^{2}-x^{2}\right)^{2}+4 y^{4}}{m^{4}-x^{2} m^{2}-y^{4}} .
$$

It was originally conjectured by H. Cohen and then proved by D. Bradley [7] and, independently, by T. Rivoal [18]. Their proof consists of reduction of (5) to a finite non-trivial combinatorial identity which can be proved on the basis of Almkvist and Granville's work [1]. Another proof of (5]) based on application of WZ-pairs was given by the authors in [9]. Since

$$
\sum_{k=1}^{\infty} \frac{k}{k^{4}-x^{2} k^{2}-y^{4}}=\sum_{n=0}^{\infty} \sum_{m=0}^{\infty}\left(\begin{array}{c}
n+m \\
n
\end{array}\right) \zeta(2 n+4 m+3) x^{2 n} y^{4 m}, \quad|x|^{2}+|y|^{4}<1,
$$

the formula (5) generates Apéry-like series for all $\zeta(2 n+4 m+3), n, m \geq 0$, convergent at the geometric rate with ratio $1 / 4$ and contains, as particular cases, both identities 2 and 47 .

In [9], the authors showed that the generating function (6) also has a much more rapidly convergent representation, namely

$$
\sum_{k=1}^{\infty} \frac{k}{k^{4}-x^{2} k^{2}-y^{4}}=\frac{1}{2} \sum_{n=1}^{\infty} \frac{(-1)^{n-1} r(n)}{n\left(\begin{array}{c}
2 n \\
n
\end{array}\right)} \frac{\prod_{m=1}^{n-1}\left(\left(m^{2}-x^{2}\right)^{2}+4 y^{4}\right)}{\prod_{m=n}^{2 n}\left(m^{4}-x^{2} m^{2}-y^{4}\right)},
$$


where

$$
r(n)=205 n^{6}-160 n^{5}+\left(32-62 x^{2}\right) n^{4}+40 x^{2} n^{3}+\left(x^{4}-8 x^{2}-25 y^{4}\right) n^{2}+10 y^{4} n+y^{4}\left(x^{2}-2\right) .
$$

The identity (7) produces accelerated series for all $\zeta(2 n+4 m+3), n, m \geq$, convergent at the geometric rate with ratio $2^{-10}$. In particular, if $x=y=0$ we get Amdeberhan-Zeilberger's series [2] for $\zeta(3)$,

$$
\zeta(3)=\frac{1}{2} \sum_{n=1}^{\infty} \frac{(-1)^{n-1}\left(205 n^{2}-160 n+32\right)}{n^{5}\left(\begin{array}{c}
2 n \\
n
\end{array}\right)^{5}} .
$$

It is worth pointing out that both identities [5] and (7) were proved in [9] by using the same Markov-WZ pair (see also [10, p. 3] for the explicit expression), but with the help of different summation formulas.

A more general form of the bivariate identity (5) for the generating function

$$
\begin{aligned}
\sum_{n=0}^{\infty} \sum_{m=0}^{\infty}\left(\begin{array}{c}
m+n \\
n
\end{array}\right) & \left(A_{0} \zeta(2 n+4 m+4)+B_{0} \zeta(2 n+4 m+3)+C_{0} \zeta(2 n+4 m+2)\right) x^{2 n} y^{4 m} \\
= & \sum_{k=1}^{\infty} \frac{A_{0}+B_{0} k+C_{0} k^{2}}{k^{4}-x^{2} k^{2}-y^{4}}, \quad|x|^{2}+|y|^{4}<1,
\end{aligned}
$$

where $A_{0}, B_{0}, C_{0}$ are arbitrary complex numbers, was proved in [9] by means of the Markov-WilfZelberger theory. More precisely, one has

$$
\sum_{k=1}^{\infty} \frac{A_{0}+B_{0} k+C_{0} k^{2}}{k^{4}-x^{2} k^{2}-y^{4}}=\sum_{n=1}^{\infty} \frac{d_{n}}{\prod_{m=1}^{n}\left(m^{4}-x^{2} m^{2}-y^{4}\right)}
$$

where

$$
\begin{aligned}
d_{n} & =\frac{(-1)^{n-1} B_{0}\left(5 n^{2}-x^{2}\right)}{2 n\left(\begin{array}{c}
2 n \\
n
\end{array}\right)} \prod_{m=1}^{n-1}\left(\left(m^{2}-x^{2}\right)^{2}+4 y^{4}\right) \\
& +\frac{(40 n+10) L_{n}+\left(35 n^{5}-35 n^{3} x^{2}+4 n\left(3 x^{4}+10 y^{4}\right)\right) L_{n-1}}{4\left(5 n^{2}-2 x^{2}\right)}
\end{aligned}
$$

and $L_{n}$ is a solution of a certain second order linear difference equation with polynomial coefficients in $n$ and $x, y$ with the initial values $L_{0}=C_{0}, L_{1}=\left(5-2 x^{2}\right) A_{0} / 15+\left(5 x^{2}-1-4\left(x^{4}+6 y^{4}\right)\right) C_{0} / 30$. If we take $A_{0}=C_{0}=0, B_{0}=1$ in (9), then $L_{n}=0$ for all $n \geq 0$ and we get the bivariate identity (5). If $B_{0}=C_{0}=0, A_{0}=1$, then we obtain

$$
\begin{aligned}
& \sum_{n=0}^{\infty} \sum_{m=0}^{\infty}\left(\begin{array}{c}
m+n \\
n
\end{array}\right) \zeta(2 n+4 m+4) x^{2 n} y^{4 m} \\
& \quad=\frac{1}{4} \sum_{n=1}^{\infty} \frac{(40 n+10) L_{n}+\left(35 n^{5}-35 x^{2} n^{3}+4 n\left(3 x^{4}+10 y^{4}\right)\right) L_{n-1}}{\left(5 n^{2}-2 x^{2}\right) \prod_{m=1}^{n}\left(m^{4}-x^{2} m^{2}-y^{4}\right)}
\end{aligned}
$$

where the sequence $L_{n}$ is defined recursively as above. A similar formula is valid for the sequence $\zeta(2 n+4 m+2)$. 
First results related to generating function identities for even zeta values belong to Leshchiner [12] who proved (in an expanded form) that for $|a|<1$,

$$
\sum_{n=0}^{\infty}\left(1-\frac{1}{2^{2 n+1}}\right) \zeta(2 n+2) a^{2 n}=\sum_{n=1}^{\infty} \frac{(-1)^{n-1}}{n^{2}-a^{2}}=\frac{1}{2} \sum_{k=1}^{\infty} \frac{1}{k^{2}\left(\begin{array}{c}
2 k \\
k
\end{array}\right)} \frac{3 k^{2}+a^{2}}{k^{2}-a^{2}} \prod_{m=1}^{k-1}\left(1-\frac{a^{2}}{m^{2}}\right) .
$$

Comparing constant terms on both sides of (10) yields

$$
\zeta(2)=3 \sum_{k=1}^{\infty} \frac{1}{k^{2}\left(\begin{array}{c}
2 k \\
k
\end{array}\right)}
$$

Since

$$
\zeta(2 n)=(-1)^{n-1} \frac{(2 \pi)^{2 n}}{2 \cdot(2 n) !} B_{2 n},
$$

where $B_{2 n} \in \mathbb{Q}$ are the Bernoulli numbers generated by the exponential generating function

$$
\frac{x}{e^{x}-1}=\sum_{n=0}^{\infty} B_{n} \frac{x^{n}}{n !}
$$

formula 10 gives Apéry-like series for even powers $\pi^{2 n}, n=1,2, \ldots$ In 2006, D. Bailey, J. Borwein and D. Bradley [5] proved another identity

$$
\sum_{n=0}^{\infty} \zeta(2 n+2) a^{2 n}=\sum_{k=1}^{\infty} \frac{1}{k^{2}-a^{2}}=3 \sum_{k=1}^{\infty} \frac{1}{\left(\begin{array}{c}
2 k \\
k
\end{array}\right)\left(k^{2}-a^{2}\right)} \prod_{m=1}^{k-1}\left(\frac{m^{2}-4 a^{2}}{m^{2}-a^{2}}\right) .
$$

It generates similar Apéry-like series for the numbers $\zeta(2 n+2)$, which are not covered by Leshchiner's result (10]. In the same paper [5], a generating function producing fast convergent series for the sequence $\pi^{2 n+4}, n=0,1,2, \ldots$, was found, which for $|a|<1$, has the form

$$
\sum_{n=0}^{\infty} \frac{(-1)^{n}\left(3^{-2 n-3}-2 B_{2 n+4}\left(2^{2 n+3}-1\right)\right) \pi^{2 n+4}}{4(2 n+4) !} a^{2 n}=\sum_{k=1}^{\infty} \frac{1}{k^{2}\left(\begin{array}{c}
2 k \\
k
\end{array}\right)\left(k^{2}-a^{2}\right)} \prod_{m=1}^{k-1}\left(1-\frac{a^{2}}{m^{2}}\right) .
$$

Here the left-hand side of (11) is a Maclaurin expansion of the function

$$
\frac{\pi a \csc (\pi a)+3 \cos (\pi a / 3)-4}{4 a^{4}} .
$$

Comparing constant terms in 111 implies that

$$
\zeta(4)=\frac{36}{17} \sum_{k=1}^{\infty} \frac{1}{k^{4}\left(\begin{array}{c}
2 k \\
k
\end{array}\right)} .
$$

The identity 11 gives a formula for $\pi^{2 n+4}$ which for $n \geq 0$ involves fewer summations then the corresponding formula generated by 10 . 
In this paper, we consider values of Dirichlet's beta function, which for $\Re s>0$ is defined by the series

$$
\beta(s)=\sum_{k=0}^{\infty} \frac{(-1)^{k}}{(2 k+1)^{s}} .
$$

It is well-known due to Euler that for odd $s, \beta(s)$ is a rational multiple of $\pi^{s}$

$$
\beta(2 n+1)=\frac{(-1)^{n} E_{2 n}}{2^{2 n+2}(2 n) !} \pi^{2 n+1}, \quad n=0,1,2, \ldots,
$$

where the integer coefficients $E_{2 n}$ are the even indexed Euler numbers defined by the exponential generating function

$$
\frac{1}{\cosh (z)}=\frac{2 e^{z}}{e^{2 z}+1}=\sum_{n=0}^{\infty} \frac{E_{n}}{n !} z^{n}=1-\frac{z^{2}}{2 !}+\frac{5 z^{4}}{4 !}-\frac{61 z^{6}}{6 !}+\ldots
$$

For even $s$, no Euler-type formula is known, and $\beta(2)$ defines the well-known Catalan's constant

$$
G:=\sum_{n=0}^{\infty} \frac{(-1)^{n}}{(2 n+1)^{2}}=0.915965594 \ldots
$$

In Sections 2, 3 we prove generating function identities producing fast convergent series for the sequences $\beta(2 n+1)$ and $\beta(2 n+2)$. In particular, we establish some analogs of identities $(10)$ and 11 for the odd powers of $\pi: \pi^{2 n+1}, n \geq 0$, and $\pi^{2 n+3}, n \geq 0$. As a consequence of our results on even beta values, we derive the following nice formula for Catalan's constant:

$$
G=\frac{1}{64} \sum_{n=1}^{\infty} \frac{(-1)^{n-1} 256^{n} q(n)}{\left(\begin{array}{c}
8 n \\
4 n
\end{array}\right)^{2}\left(\begin{array}{c}
2 n \\
n
\end{array}\right) n^{3}(2 n-1)(4 n-1)^{2}(4 n-3)^{2}},
$$

where

$$
q(n)=419840 n^{6}-915456 n^{5}+782848 n^{4}-332800 n^{3}+73256 n^{2}-7800 n+315 .
$$

It can be considered as an analog of Amdeberhan-Zeilberger's series 8 for $\zeta(3)$, since the series on the right-hand side of 13 converges exponentially with ratio $2^{-10}$.

\section{Apéry-like series for $\beta(2 n+1)$ and $\beta(2 n+3)$.}

We start by recalling several definitions and known facts related to the Markov-Wilf-Zeilberger theory (see [14, 15, 16]). A function $H(n, k)$, in the integer variables $n$ and $k$, is called hypergeometric or closed form $(C F)$ if the quotients

$$
\frac{H(n+1, k)}{H(n, k)} \quad \text { and } \quad \frac{H(n, k+1)}{H(n, k)}
$$


are both rational functions of $n$ and $k$. A hypergeometric function that can be written as a ratio of products of factorials is called pure-hypergeometric. A pair of CF functions $F(n, k)$ and $G(n, k)$ is called a WZpair if

$$
F(n+1, k)-F(n, k)=G(n, k+1)-G(n, k) .
$$

A $P$-recursive function is a function that satisfies a linear recurrence relation with polynomial coefficients. If for a given hypergeometric function $H(n, k)$, there exists a polynomial $P(n, k)$ in $k$ of the form

$$
P(n, k)=a_{0}(n)+a_{1}(n) k+\cdots+a_{L}(n) k^{L},
$$

for some non-negative integer $L$, and P-recursive functions $a_{0}(n), \ldots, a_{L}(n)$ such that

$$
F(n, k):=H(n, k) P(n, k)
$$

satisfies (14) with some function $G$, then a pair $(F, G)$ is called a Markov-WZ pair associated with the kernel $H(n, k)$ (MWZ-pair for short). We call $G(n, k)$ an $M W Z$ mate of $F(n, k)$. If $L=0$, then $(F, G)$ is simply a WZ-pair.

In 2005, M. Mohammed [15] showed that for any pure-hypergeometric kernel $H(n, k)$, there exists a non-negative integer $L$ and a polynomial $P(n, k)$ as above such that $F(n, k)=H(n, k) P(n, k)$ has an MWZ mate $G(n, k)=F(n, k) Q(n, k)$, where $Q(n, k)$ is a ratio of two P-recursive functions. Paper [16] is accompanied by the Maple package MarkovWZ which, for a given $H(n, k)$ outputs the polynomial $P(n, k)$ and the $G(n, k)$ as above.

From relation (14) we get the following summation formulas.

Proposition A. [15, Theorem 2(b)] Let $(F, G)$ be an MWZ-pair. If $\lim _{n \rightarrow \infty} F(n, k)=0$ for every $k \geq 0$, then

$$
\sum_{k=0}^{\infty} F(0, k)-\lim _{k \rightarrow \infty} \sum_{n=0}^{\infty} G(n, k)=\sum_{n=0}^{\infty} G(n, 0),
$$

whenever both sides converge.

Proposition B. [15, Cor. 2] Let $(F, G)$ be an MWZ-pair. If $\lim _{k \rightarrow \infty} \sum_{n=0}^{\infty} G(n, k)=0$, then

$$
\sum_{k=0}^{\infty} F(0, k)=\sum_{n=0}^{\infty}(F(n, n)+G(n, n+1)),
$$

whenever both sides converge.

Formulas (15), 16] with an appropriate choice of MWZ-pairs can be used to convert a given hypergeometric series into a different rapidly converging one.

As usual, let $(\lambda)_{\nu}$ be the Pochhammer symbol (or the shifted factorial) defined by

$$
(\lambda)_{\nu}=\frac{\Gamma(\lambda+\nu)}{\Gamma(\lambda)}= \begin{cases}1, & \nu=0 \\ \lambda(\lambda+1) \ldots(\lambda+\nu-1), & \nu \in \mathbb{N} .\end{cases}
$$

In 1979, D. Leshchiner [12] proved accelerated series for the values $\beta(2 n+1)$ in the spirit of Apéry's series (1). Namely, he showed that

$$
\beta(2 n+1)=\sum_{k=0}^{\infty} \frac{\left(\begin{array}{c}
2 k \\
k
\end{array}\right)}{16^{k}} \sum_{\nu=0}^{n} \frac{(-1)^{n-\nu} A_{\nu}}{(2 k+1)^{2 \nu+1}} f_{k+1}^{n-\nu}, \quad n=0,1,2, \ldots
$$


where $f_{k}^{0}=1, f_{k}^{r}=0$ if $r<0$ or $r \geq k$,

$$
f_{k}^{r}=\sum_{0<l_{1}<\ldots<l_{r}<k} \prod_{j=1}^{r} \frac{1}{\left(2 l_{j}-1\right)^{2}}, \quad 1 \leq r \leq k-1,
$$

and

$$
A_{\nu}= \begin{cases}3 / 4 & \text { if } \quad \nu=0 \\ 1 & \text { if } \quad \nu>0\end{cases}
$$

Formulas 12, 17] give the following Apéry-like series for odd powers of $\pi$ :

$$
\begin{gathered}
\pi=3 \sum_{k=0}^{\infty} \frac{\left(\begin{array}{c}
2 k \\
k
\end{array}\right)}{16^{k}(2 k+1)}, \\
\pi^{3}=32 \sum_{k=0}^{\infty} \frac{\left(\begin{array}{c}
2 k \\
k
\end{array}\right)}{16^{k}(2 k+1)^{3}}-24 \sum_{k=0}^{\infty} \frac{\left(\begin{array}{c}
2 k \\
k
\end{array}\right)}{16^{k}(2 k+1)} \sum_{m=0}^{k-1} \frac{1}{(2 m+1)^{2}}, \\
\pi^{5}=\frac{1536}{5} \sum_{k=0}^{\infty} \frac{\left(\begin{array}{c}
2 k \\
k
\end{array}\right)}{16^{k}(2 k+1)^{5}}-\frac{1536}{5} \sum_{k=0}^{\infty} \frac{\left(\begin{array}{c}
2 k \\
k
\end{array}\right)}{16^{k}(2 k+1)^{3}} \sum_{m=0}^{k-1} \frac{1}{(2 m+1)^{2}} \\
+\frac{1152}{5} \sum_{k=0}^{\infty} \frac{\left(\begin{array}{c}
2 k \\
k
\end{array}\right)}{16^{k}(2 k+1)} \sum_{m=0}^{k-1} \sum_{l=0}^{k-1} \frac{1}{(2 m+1)^{2}(2 l+1)^{2}} .
\end{gathered}
$$

Using the generating function for odd beta values

$$
\sum_{n=0}^{\infty} \beta(2 n+1) a^{2 n}=\frac{1}{2} \sum_{k=0}^{\infty} \frac{(-1)^{k}(k+1 / 2)}{(k+1 / 2)^{2}-a^{2} / 4}
$$

it is easily seen that formulas (17) are generated by the following identity.

Proposition 1 For any complex a with $|a|<1$ we have

$$
\sum_{n=0}^{\infty} \beta(2 n+1) a^{2 n}=\sum_{n=0}^{\infty} \frac{\left(\begin{array}{c}
2 n \\
n
\end{array}\right)}{4^{2 n+1}(2 n+1)} \frac{3(2 n+1)^{2}+a^{2}}{(2 n+1)^{2}-a^{2}} \prod_{m=0}^{n-1}\left(1-\frac{a^{2}}{(2 m+1)^{2}}\right) .
$$

Proof. Identity (19) can easily be proved by the Markov-Wilf-Zeilberger method. Taking the kernel

$$
H(n, k)=\frac{(-1)^{k} k !(n+k+1 / 2)}{(k+2 n) !\left((n+k+1 / 2)^{2}-a^{2} / 4\right)}
$$

and applying the Maple package MarkovWZ we get that

$$
F(n, k)=\frac{2(-1)^{k} k !(2 n+2 k+1)}{(k+2 n) !\left((2 n+2 k+1)^{2}-a^{2}\right)}\left(\frac{1+a}{2}\right)_{n}\left(\frac{1-a}{2}\right)_{n}
$$


and

$$
G(n, k)=\frac{(-1)^{k} k !\left(3(2 n+1)^{2}+a^{2}+4 k(2+4 n+k)\right)}{2(k+2 n+1) !\left((2 n+2 k+1)^{2}-a^{2}\right)}\left(\frac{1+a}{2}\right)_{n}\left(\frac{1-a}{2}\right)_{n}
$$

give a WZ-pair, i.e.,

$$
F(n+1, k)-F(n, k)=G(n, k+1)-G(n, k) .
$$

Now by Proposition A, we get

$$
\sum_{k=0}^{\infty} F(0, k)=\sum_{n=0}^{\infty} G(n, 0)
$$

which implies 19 .

Lemma 1 For any complex a,

$$
\sin \frac{\pi a}{6}=\frac{a}{2} \sum_{n=0}^{\infty} \frac{\left(\begin{array}{c}
2 n \\
n
\end{array}\right)}{16^{n}(2 n+1)} \prod_{m=0}^{n-1}\left(1-\frac{a^{2}}{(2 m+1)^{2}}\right) .
$$

Proof. Applying the transformation (see [6, §2.8]) in terms of the Gauss hypergeometric function

$$
\sin a z=a \sin z \cdot{ }_{2} F_{1}\left(\frac{1+a}{2}, \frac{1-a}{2} ; \frac{3}{2} ; \sin ^{2} z\right)
$$

with $z=\pi / 6$ we get the desired representation.

Remark. Formula $(20)$ can be considered as an expansion of the function $\sin (\pi a / 6)$ into Newton's interpolation series with interpolation points at zero and odd integers.

Theorem 1 Let a be a complex number, distinct from an odd integer. Then

$$
\frac{\pi}{4 a^{2} \cos (\pi a / 2)}-\frac{3}{2 a^{3}} \sin \frac{\pi a}{6}=\sum_{n=0}^{\infty} \frac{\left(\begin{array}{c}
2 n \\
n
\end{array}\right)}{16^{n}(2 n+1)\left((2 n+1)^{2}-a^{2}\right)} \prod_{m=0}^{n-1}\left(1-\frac{a^{2}}{(2 m+1)^{2}}\right) .
$$

If $|a|<1$, then the left-hand side of 21] has the following series expansion:

$$
\sum_{n=0}^{\infty} \frac{(-1)^{n+1}\left((2 n+3) 9^{n+1} E_{2 n+2}-1\right) \pi^{2 n+3}}{4^{n+2} 9^{n+1}(2 n+3) !} a^{2 n}=\frac{7 \pi^{3}}{216}+\frac{253 \pi^{5}}{77760} a^{2}+\ldots
$$

In particular, we get

$$
\begin{gathered}
\pi^{3}=\frac{216}{7} \sum_{n=0}^{\infty} \frac{\left(\begin{array}{c}
2 n \\
n
\end{array}\right)}{16^{n}(2 n+1)^{3}} \\
\pi^{5}=\frac{77760}{253} \sum_{n=0}^{\infty} \frac{\left(\begin{array}{c}
2 n \\
n
\end{array}\right)}{16^{n}(2 n+1)^{5}}-\frac{77760}{253} \sum_{n=0}^{\infty} \frac{\left(\begin{array}{c}
2 n \\
n
\end{array}\right)}{16^{n}(2 n+1)^{3}} \sum_{m=0}^{n-1} \frac{1}{(2 m+1)^{2}} .
\end{gathered}
$$


Proof. By Proposition 1 and Lemma 1 we get

$$
\sum_{n=0}^{\infty} \beta(2 n+1) a^{2 n}=\frac{3}{2 a} \sin \frac{\pi a}{6}+a^{2} \sum_{n=0}^{\infty} \frac{\left(\begin{array}{c}
2 n \\
n
\end{array}\right)}{16^{n}(2 n+1)\left((2 n+1)^{2}-a^{2}\right)} \prod_{m=0}^{n-1}\left(1-\frac{a^{2}}{(2 m+1)^{2}}\right) .
$$

On the other hand, by $[18)$, we have

$$
4 \cdot \sum_{n=0}^{\infty} \beta(2 n+1) a^{2 n}=g\left(\frac{1-a}{2}\right)+g\left(\frac{1+a}{2}\right)
$$

where $g(z)=\sum_{k=0}^{\infty}(-1)^{k} /(k+z)$. Using the reflection formula (see [6, §1.8])

$$
g(z)+g(1-z)=\frac{\pi}{\sin (\pi z)}
$$

we get

$$
\sum_{n=0}^{\infty} \beta(2 n+1) a^{2 n}=\frac{\pi}{4 \cos (\pi a / 2)},
$$

from which formula (21) follows. Expanding the left-hand side of (21), by (12), we get the required expansion.

Comparing (21) and (19) we can easily see that these identities produce different series for the odd powers of $\pi$. The series generated by (21) are simpler in that sense they involve fewer summations then the corresponding series generated by (19). Moreover, the formulas obtained here lead to some non-trivial redundancy relations that can be written down explicitly by comparing (17) and the corresponding series for $\beta(2 n+1), n \geq 1$, given by 21 . As an example, comparing series for $\pi^{3}$ yields the following redundancy formula:

$$
\sum_{n=0}^{\infty} \frac{\left(\begin{array}{c}
2 n \\
n
\end{array}\right)}{16^{n}(2 n+1)} \sum_{m=0}^{n-1} \frac{1}{(2 m+1)^{2}}=\frac{1}{21} \sum_{n=0}^{\infty} \frac{\left(\begin{array}{c}
2 n \\
n
\end{array}\right)}{16^{n}(2 n+1)^{3}} .
$$

\section{Generating function identities for even beta values.}

In this section we derive series acceleration formulae for even beta values by application of the MarkovWZ method.

Theorem 2 Let $a$ and $d$ be complex numbers such that $a^{2} \neq(d+n)^{2}, n=0,1,2, \ldots$, and distinct from zero and negative integers. Then

$$
\sum_{k=0}^{\infty} \frac{(-1)^{k}}{(k+d)^{2}-a^{2}}=\frac{1}{2} \sum_{n=0}^{\infty} \frac{(1+a)_{n}(1-a)_{n}\left(3 n^{2}+2 n(2 d+1)+d^{2}+2 d+a^{2}\right)}{(d)_{2 n+2}\left((n+d)^{2}-a^{2}\right)} .
$$


Proof. Considering the kernel

$$
H(n, k)=\frac{(-1)^{k}(d)_{k}(n+k+d)}{(d)_{2 n+k+1}\left((n+k+d)^{2}-a^{2}\right)}
$$

we get that

$$
F(n, k)=\frac{(-1)^{k}(d)_{k}(n+k+d)(1+a)_{n}(1-a)_{n}}{(d)_{2 n+k+1}\left((n+k+d)^{2}-a^{2}\right)}
$$

and

$$
\begin{aligned}
G(n, k) & =\frac{(-1)^{k}(d)_{k}(1+a)_{n}(1-a)_{n}}{2(d)_{2 n+k+2}\left((n+k+d)^{2}-a^{2}\right)} \times \\
& \times\left(3 n^{2}+2 n(2 d+1)+d^{2}+2 d+a^{2}+k^{2}+k(2 d+4 n+2)\right)
\end{aligned}
$$

give a WZ-pair, and by Proposition A, we obtain

$$
\sum_{k=0}^{\infty} F(0, k)=\sum_{n=0}^{\infty} G(n, 0)
$$

implying 22].

In particular, from 22 if $d=1$ we get Leshchiner's identity 10 . If $d=1 / 2$ we get the following identity generating accelerated series for even beta values.

Corollary 1 Let a be a complex number with $|a|<1$. Then

$$
\sum_{n=0}^{\infty} \beta(2 n+2) a^{2 n}=\frac{1}{8} \sum_{n=1}^{\infty} \frac{16^{n}\left(12 n^{2}-8 n+1+a^{2}\right)}{\left(\begin{array}{c}
4 n \\
2 n
\end{array}\right)\left(\begin{array}{c}
2 n \\
n
\end{array}\right) n^{2}\left((2 n-1)^{2}-a^{2}\right)} \prod_{m=1}^{n-1}\left(1-\frac{a^{2}}{4 m^{2}}\right) .
$$

In particular, if $a=0$ we get

$$
G=\frac{1}{8} \sum_{n=1}^{\infty} \frac{16^{n}(6 n-1)}{\left(\begin{array}{c}
4 n \\
2 n
\end{array}\right)\left(\begin{array}{c}
2 n \\
n
\end{array}\right) n^{2}(2 n-1)}
$$

Theorem 3 Let a be a complex number with $|a|<1$. Then

$$
\sum_{n=0}^{\infty} \beta(2 n+2) a^{2 n}=\frac{1}{64} \sum_{n=1}^{\infty} \frac{(-1)^{n-1} 256^{n}\left(40 n^{2}-24 n+3-a^{2}\right)}{\left(\begin{array}{c}
4 n \\
2 n
\end{array}\right)^{2}\left(\begin{array}{c}
2 n \\
n
\end{array}\right) n^{3}(2 n-1)} \frac{\prod_{m=1}^{n-1}\left(1-a^{2} /\left(4 m^{2}\right)\right)}{\prod_{m=1}^{2 n}\left(1-a^{2} /(2 m-1)^{2}\right)} .
$$

In particular,

$$
G=\frac{1}{64} \sum_{n=1}^{\infty} \frac{(-1)^{n-1} 256^{n}\left(40 n^{2}-24 n+3\right)}{\left(\begin{array}{c}
4 n \\
2 n
\end{array}\right)^{2}\left(\begin{array}{c}
2 n \\
n
\end{array}\right) n^{3}(2 n-1)}
$$

Proof. Starting with the kernel

$$
H(n, k)=(-1)^{k} \frac{\left(\frac{1+a}{2}\right)_{k}\left(\frac{1-a}{2}\right)_{k}}{\left(\frac{1-a}{2}\right)_{2 n+k+1}\left(\frac{1+a}{2}\right)_{2 n+k+1}}
$$


and applying the Maple package MarkovWZ [16], we see that

$$
F(n, k)=\frac{(-1)^{n+k}\left(\frac{1+a}{2}\right)_{k}\left(\frac{1-a}{2}\right)_{k}\left(1+\frac{a}{2}\right)_{n}\left(1-\frac{a}{2}\right)_{n}(2 n) !}{\left(\frac{1-a}{2}\right)_{2 n+k+1}\left(\frac{1+a}{2}\right)_{2 n+k+1}}
$$

and

$$
\begin{aligned}
G(n, k) & =\frac{(-1)^{n+k}\left(\frac{1+a}{2}\right)_{k}\left(\frac{1-a}{2}\right)_{k}\left(1+\frac{a}{2}\right)_{n}\left(1-\frac{a}{2}\right)_{n}(2 n) !}{8\left(\frac{1-a}{2}\right)_{2 n+k+2}\left(\frac{1+a}{2}\right)_{2 n+k+2}} \times \\
& \times\left(40 n^{2}+56 n+19-a^{2}+24 n k+16 k+4 k^{2}\right)
\end{aligned}
$$

is a Markov-WZ pair. By using Proposition A, we get

$$
\sum_{k=0}^{\infty} F(0, k)=\sum_{n=0}^{\infty} G(n, 0)
$$

which implies the required statement.

The generalized identities of Corollary 1 and Theorem 3 generate fast convergent series for $\beta(2 n+2)$ convergent at a geometric rate with ratio 1/4. Formula 23 was earlier found by Lupas in [13]. The next corollary gives much faster convergent series at the geometric rate with ratio $2^{2} / 3^{6}=4 / 729=0.005 \ldots$.

Corollary 2 Let a be a complex number with $|a|<1$. Then

$$
\sum_{k=0}^{\infty} \beta(2 k+2) a^{2 k}=\frac{1}{16} \sum_{n=1}^{\infty} \frac{256^{n} p(n, a)}{n^{3}(2 n-1)^{3}\left(\begin{array}{c}
6 n \\
3 n
\end{array}\right)\left(\begin{array}{c}
6 n \\
4 n
\end{array}\right)\left(\begin{array}{c}
4 n \\
2 n
\end{array}\right)} \frac{\prod_{m=1}^{n-1}\left(1-a^{2} /\left(4 m^{2}\right)\right)}{\prod_{m=n}^{3 n}\left(1-a^{2} /(2 m-1)^{2}\right)}
$$

where $p(n, a)=580 n^{4}-764 n^{3}+344 n^{2}-61 n+15 / 4-a^{2}\left(18 n^{2}-15 n+4-a^{2} / 4\right)$.

In particular,

$$
G=\frac{1}{64} \sum_{n=1}^{\infty} \frac{256^{n}\left(580 n^{2}-184 n+15\right)}{n^{3}(2 n-1)\left(\begin{array}{c}
6 n \\
3 n
\end{array}\right)\left(\begin{array}{c}
6 n \\
4 n
\end{array}\right)\left(\begin{array}{c}
4 n \\
2 n
\end{array}\right)}
$$

Proof. By applying Proposition B to the MWZ pair from the proof of Theorem 3 we get

$$
\sum_{k=0}^{\infty} F(0, k)=\sum_{n=0}^{\infty}(F(n, n)+G(n, n+1))
$$

or

$$
\sum_{k=0}^{\infty} \beta(2 k+2) a^{2 k}=\frac{1}{32} \sum_{n=0}^{\infty} \frac{\left(\frac{1+a}{2}\right)_{n}\left(\frac{1-a}{2}\right)_{n}\left(1+\frac{a}{2}\right)_{n}\left(1-\frac{a}{2}\right)_{n}(2 n) ! p(n+1, a)}{\left(\frac{1+a}{2}\right)_{3 n+3}\left(\frac{1-a}{2}\right)_{3 n+3}}
$$

where $p(n, a)$ is defined as above. After simplifying and replacing Pochhammer's symbols by binomial coefficients we get the desired identity.

The next theorem gives even much faster convergent series at the geometric rate with ratio $2^{-10}$. 
Theorem 4 Let a be a complex number with $|a|<1$. Then

$$
\sum_{k=0}^{\infty} \beta(2 k+2) a^{2 k}=\frac{1}{64} \sum_{n=1}^{\infty} \frac{(-1)^{n-1} 256^{n} q(n, a)}{\left(\begin{array}{c}
8 n \\
4 n
\end{array}\right)^{2}\left(\begin{array}{c}
2 n \\
n
\end{array}\right) n^{3}(2 n-1)(4 n-1)^{2}(4 n-3)^{2}} \frac{\prod_{m=1}^{n-1}\left(1-a^{2} /\left(4 m^{2}\right)\right)}{\prod_{m=2 n-1}^{4 n}\left(1-a^{2} /(2 m-1)^{2}\right)},
$$

where

$$
\begin{aligned}
& q(n, a)=419840 n^{6}-915456 n^{5}+n^{4}\left(782848-15872 a^{2}\right)-n^{3}\left(332800-25600 a^{2}\right) \\
& +n^{2}\left(73256-14992 a^{2}+232 a^{4}\right)-n\left(7800-3760 a^{2}+184 a^{4}\right)+315-359 a^{2}+45 a^{4}-a^{6} .
\end{aligned}
$$

In particular,

$$
G=\frac{1}{64} \sum_{n=1}^{\infty} \frac{(-1)^{n-1} 256^{n} q(n)}{\left(\begin{array}{c}
8 n \\
4 n
\end{array}\right)^{2}\left(\begin{array}{c}
2 n \\
n
\end{array}\right) n^{3}(2 n-1)(4 n-1)^{2}(4 n-3)^{2}}
$$

where

$$
q(n)=419840 n^{6}-915456 n^{5}+782848 n^{4}-332800 n^{3}+73256 n^{2}-7800 n+315 .
$$

Proof. We first observe that the generating function of even beta values can be written in the form

$$
\begin{aligned}
\sum_{k=0}^{\infty} \beta(2 k+2) a^{2 k} & =\sum_{n=0}^{\infty} \frac{(-1)^{n}}{(2 n+1)^{2}-a^{2}}=\sum_{k=0}^{\infty}\left(\frac{1}{(4 k+1)^{2}-a^{2}}-\frac{1}{(4 k+3)^{2}-a^{2}}\right) \\
& =\frac{1}{32} \sum_{k=0}^{\infty} \frac{2 k+1}{\left((k+1 / 4)^{2}-a^{2} / 16\right)\left((k+3 / 4)^{2}-a^{2} / 16\right)}
\end{aligned}
$$

Define the function

$$
H(n, k)=\frac{\left(\frac{1+a}{4}\right)_{k}\left(\frac{1-a}{4}\right)_{k}\left(\frac{3+a}{4}\right)_{k}\left(\frac{3-a}{4}\right)_{k}(n+1+2 k)}{\left(\frac{1+a}{4}\right)_{n+k+1}\left(\frac{1-a}{4}\right)_{n+k+1}\left(\frac{3+a}{4}\right)_{n+k+1}\left(\frac{3-a}{4}\right)_{n+k+1}} .
$$

Then using the Maple package MarkovWZ we obtain that

$$
F(n, k)=\frac{(-1)^{n}}{16^{n}}(2 n+1) !\left(1+\frac{a}{2}\right)_{n}\left(1-\frac{a}{2}\right)_{n} H(n, k)
$$

and

$$
G(n, k)=\frac{(-1)^{n}(2 n) ! H(n, k)}{16^{n+1}(n+1+2 k)}\left(1+\frac{a}{2}\right)_{n}\left(1-\frac{a}{2}\right)_{n}\left(40 n^{2}+56 n+19-a^{2}+16 k(k+3 n+2)\right)
$$

is a Markov-WZ pair corresponding to the kernel $H(n, k)$. Now by Proposition B, we have

$$
\sum_{n=0}^{\infty}(F(n, n)+G(n, n+1))=\sum_{k=0}^{\infty} F(0, k)=\sum_{k=0}^{\infty} H(0, k)=32 \sum_{k=0}^{\infty} \beta(2 k+2) a^{2 k}
$$


or

$$
\begin{gathered}
\sum_{k=0}^{\infty} \beta(2 k+2) a^{2 k} \\
=\frac{1}{32} \sum_{n=0}^{\infty} \frac{(-1)^{n}(2 n) !}{16^{n+1}} \frac{\left(1+\frac{a}{2}\right)_{n}\left(1-\frac{a}{2}\right)_{n}\left(\frac{1+a}{4}\right)_{n}\left(\frac{1-a}{4}\right)_{n}\left(\frac{3+a}{4}\right)_{n}\left(\frac{3-a}{4}\right)_{n}}{\left(\frac{1+a}{4}\right)_{2 n+2}\left(\frac{1-a}{4}\right)_{2 n+2}\left(\frac{3+a}{4}\right)_{2 n+2}\left(\frac{3-a}{4}\right)_{2 n+2}} q(n+1, a),
\end{gathered}
$$

where $q(n, a)$ is defined as above. After simplifying and replacing Pochhammer's symbols by binomial coefficients we get the required identity.

Note that application of Proposition A to the Markov-WZ pair found above recovers Theorem 3

In conclusion we note that the different approaches described in the introduction for studying and finding various Apéry-like series for zeta values are applicable to the Dirichlet beta function as well. Hence, it would be of interest to find more general identities for beta values by means of bivariate generating functions as it was done in the case of the zeta function (see for instance identities (5), (7)).

\section{Acknowledgements}

Both authors thank the Institut de Hautes Études Scientifiques, Bures-sur-Yvette, France, where this research was carried out for hospitality and excellent working conditions.

\section{References}

[1] G. Almkvist, A. Granville, Borwein and Bradley's Apéry-like formulae for $\zeta(4 n+3)$, Experiment. Math. 8 (1999), 197-203.

[2] T. Amdeberhan, D. Zeilberger, Hypergeometric series acceleration via the WZ method, Electron. J. Combinatorics 4(2) (1997), \#R3.

[3] J. M. Borwein, D. M. Bradley, Empirically determined Apéry-like formulae for $\zeta(4 n+3)$, Experiment. Math. 6 (1997), no. 3, 181-194.

[4] J. M. Borwein, D. M. Bradley, Searching symbolically for Apéry-like formulae for values of the Riemann zeta function, SIGSAM Bulletin of Algebraic and Symbolic Manipulation, Association of Computing Machinery, 30 (1996), no. 2, 2-7.

[5] D. H. Bailey, J. M. Borwein, D. M. Bradley, Experimental determination of Apéry-like identities for $\zeta(2 n+2)$, Experiment. Math. 15 (2006), no. 3, 281-289.

[6] H. Bateman and A. Erdélyi, Higher Transcendental Functions, Vol. 1, McGraw-Hill, New York, 1953.

[7] D. M. Bradley, Hypergeometric functions related to series acceleration formulas, Contemporary Math. 457 (2008), 113-125.

[8] Kh. Hessami Pilehrood, T. Hessami Pilehrood, Generating function identities for $\zeta(2 n+2), \zeta(2 n+3)$ via the WZ-method, Electron. J. Combin. 15 (2008), no. 1, Research Paper 35, 9 pp. 
[9] Kh. Hessami Pilehrood, T. Hessami Pilehrood, Simultaneous generation for zeta values by the Markov-WZ method, Discrete Math. Theor. Comput. Sci. 10 (2008), no. 3, 115-123.

[10] Kh. Hessami Pilehrood, T. Hessami Pilehrood, A q-analog of the Bailey-Borwein-Bradley identity, arXiv:0903.2843 [math.NT]

[11] M. Koecher, Letter (German), Math. Intelligencer, 2 (1979/1980), no. 2, 62-64.

[12] D. Leshchiner, Some new identities for $\zeta(k)$, J. Number Theory, 13 (1981), 355-362.

[13] A. Lupas, Formulae for some classical constants, In Proceedings of ROGER-2000. 2000. http://www.lacim.uqam.ca//plouffe/articles/alupas1.pdf

[14] A. A. Markoff, Mémoiré sur la transformation de séries peu convergentes en séries tres convergentes, Mém. de l'Acad. Imp. Sci. de St. Pétersbourg, t. XXXVII, No.9 (1890), 18pp. Available at http://www.math.mun.ca/ $/$ sergey/Research/History/Markov/markov1890.html

[15] M. Mohammed, Infinite families of accelerated series for some classical constants by the MarkovWZ method, Discrete Mathematics and Theoretical Computer Science 7 (2005), 11-24.

[16] M. Mohammed, D. Zeilberger, The Markov-WZ method, Electronic J. Combinatorics 11 (2004), \#R53.

[17] A. van der Poorten, A proof that Euler missed... Apéry's proof of the irrationality of $\zeta(3)$. An informal report, Math. Intelligencer 1 (1978/79), no. 4, 195-203.

[18] T. Rivoal, Simultaneous generation of Koecher and Almkvist-Granville's Apéry-like formulae, Experiment. Math., 13 (2004), 503-508. 